Results European American SLE patients with high disease activity were differentiated from patients with low disease activity by reduced frequencies of peripheral $\mathrm{B}$ cells, specifically naïve $B$ cells (CD27-IgD+CD24 lo) $(p=0.0101)$ and double negative $B$ cells $(C D 27-\operatorname{IgD}-)(p=0.0220)$, while African American patients with high disease activity had elevated frequencies of memory $B$ cells (CD27 $+\operatorname{IgD}$ CD $38+)$ $(\mathrm{p}<0.05)$ compared to patients with low disease activity. Several cell subsets had increased expression of activation markers during high disease activity including $B$ cells $(\mathrm{p}=0.0350)$ and plasmacytoid dendritic cells ( $\mathrm{pDCs}$ ) $(\mathrm{p}=0.0435)$ in European Americans (figure 1A), and neutrophils $(p<0.05)$, pDCs $(p=0.005)$, CD8 +T Cells $(p=0.0003)$ and NKT cells $(p=0.0033)$ in African Americans (figure 1B). Following whole blood stimulation with IFN, African American high disease activity patients were distinguished by reduced ability to activate pSTAT5 in almost all major cell populations $(\mathrm{p}<0.05)$, and pSTAT3 in monocytes $(p=0.0157)$, granulocytes $(p=0.01)$ and $B$ cells $(p=0.0409)$ compared to low disease activity patients and controls, possibly due to higher basal levels of activation (figure 1). Further, African American patients with high disease activity had significantly elevated cytokine production at baseline compared to healthy controls and European American SLE patients that translated to a reduced fold change in soluble mediators following stimulation $(\mathrm{p}<0.01)$.

Conclusions Our results support a model where race influences heightened SLE disease activity mechanisms with alterations in B cell signaling, and greater dysregulation in phospho-signaling and pro-inflammatory soluble mediators observed in African American patients.

Funding Source(s): NIH (U19AI082714, U19AI082719, U54GM104938, P30GM103510, U01AI101934)

\section{PHENOTYPIC SUBGROUPS IN SYSTEMIC LUPUS ERYTHEMATOSUS: AN EXPLORATORY CLUSTER ANALYSIS}

Amanda M Eudy*, Jennifer L Rogers, David S Pisetsky, Lisa G Criscione-Schreiber, Ravyn Njagu, Rebecca E Sadun, Kai Sun, Jayanth Doss, Megan EB Clowse. Duke University

\subsection{6/lupus-2019-Ism.288}

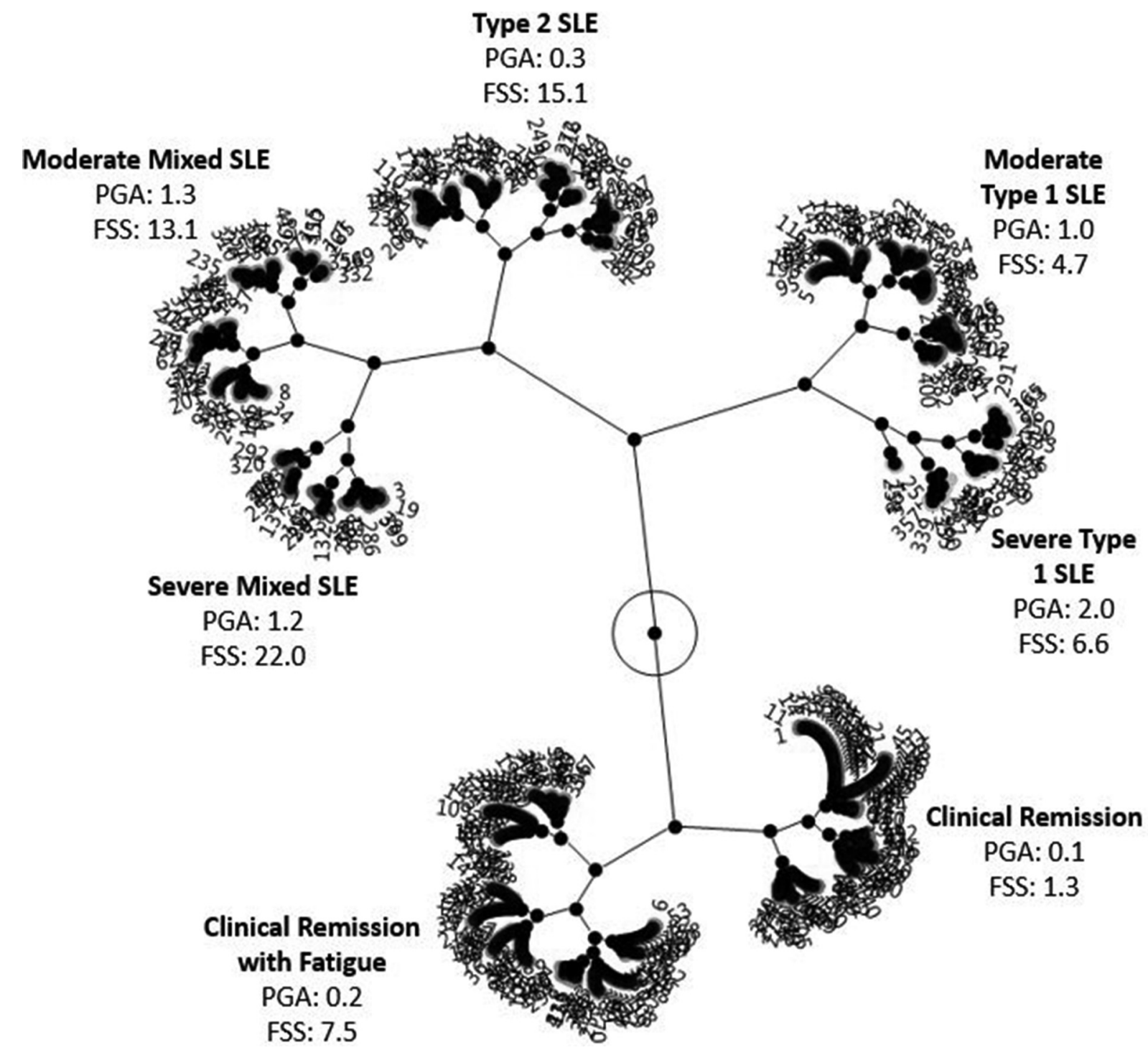

Abstract 288 Figure 1 Clustering of SLE patients by PGA and fibromyalgia severity score (FSS) 
Background Lupus is a complex, heterogeneous disease. We have developed a conceptual model to characterize SLE activity using two dimensions: Type 1 SLE includes active inflammatory manifestations of SLE, including arthritis, nephritis, and rashes; Type 2 SLE includes fatigue, myalgia, mood disturbance, and cognitive dysfunction, which can persist in the absence of inflammatory findings. We have grouped SLE patients into phenotypic clusters based on the extent of Type 1 and 2 SLE features.

Methods Consecutive SLE patients meeting ACR or SLICC criteria in a university rheumatology clinic were included. Patients completed the Systemic Lupus Activity Questionnaire (SLAQ) and the ACR Fibromyalgia (FM) Diagnostic Criteria. The Fibromyalgia Severity Score (FSS) is the sum of the widespread pain (0-19) and symptom severity (0-9) scores. The physicians global assessment (PGA) was also recorded. Patients were grouped into clusters based on PGA (Type I SLE) and FSS (Type 2 SLE) using hierarchical clustering with Wards minimum variance method. Differences were estimated by ANOVA and Fishers exact test.

Results The 419 SLE patients (92\% female; mean age: 45 years) were classified into 7 clusters (figure 1).

Minimal SLE: Clinical Remission $(n=85)$ : minimal Type 1 and 2 SLE.

Clinical Remission with Fatigue $(n=113)$ : minimal Type 1 and mild Type 2 symptoms of fatigue (70\%) and waking unrefreshed $(58 \%)$.

Predominantly Type 1: Moderate Type 1 SLE $(n=56)$ : active Type 1 SLE (43\% arthritis, 55\% anti-dsDNA+) and minimal Type 2 symptoms.

Severe Type 1 SLE $(n=31)$ : severe Type 1 SLE, with active nephritis (39\%), arthritis, new rashes, dsDNA+, and low C3/ C4, with mild Type 2 symptoms, primarily fatigue (61\%).

Predominantly Type 2: Type 2 SLE $(n=58)$ : minimal Type 1 SLE and significant Type 2 symptoms including high widespread pain scores, fatigue (97\%), waking unrefreshed (86\%), depression (67\%), cognitive dysfunction (59\%).

Mixed SLE: Moderate Mixed SLE $(n=52)$ : active Type 1 SLE (69\% arthritis, 35\% anti-dsDNA+) and active Type 2 SLE, with moderate widespread pain scores, fatigue (90\%), waking unrefreshed (96\%), forgetfulness (52\%), and depression (49\%).

Severe Mixed SLE $(n=24)$ : active Type 1 SLE $(54 \%$ arthritis, $25 \%$ proteinuria) combined with severe Type 2 SLE, with high widespread pain scores, depression (86\%), fatigue (100\%), and waking unrefreshed (100\%).

Conclusions Patient-reported measures can be identifying distinct clusters of patients with higher and lower levels of Type 1 and 2 SLE features. We have already found this approach useful in direct clinical care and are working to identify immunologic differences between clusters and optimal management protocols.

Funding Source(s): None

\section{9 COMPARISON OF ACR 1982/1997 AND EULAR/ACR CLASSIFICATION CRITERIA FOR SYSTEMIC LUPUS ERYTHEMATOSUS IN TWO MULTIETHNIC COHORTS}

${ }^{1}$ Guillermo J Pons-Estel ${ }^{*},{ }^{2}$ Manuel F Ugarte-Gil, ${ }^{3}$ Guillermina B Harvey, ${ }^{3}$ Daniel Wojdyla, ${ }^{4}$ Russell Griffin, ${ }^{5}$ Verónica Saurit, ${ }^{6}$ Enrique Soriano, ${ }^{7}$ Eloisa Bonfa, ${ }^{8}$ Loreto Massardo, ${ }^{9}$ Mario H Cardiel, ${ }^{10}$ Luis M Vila, ${ }^{11}$ Graciela S Alarcón, ${ }^{12}$ Bernardo Pons-Estel. 'Grupo Oroño Centro Regional de Enfermedades Autoinmunes y Reumáticas (GO-CREAR); ${ }^{2}$ Universidad Cientifica del Sur, ${ }^{3}$ Facultad de Ciencias Económicas y Estadistica, Universidad Nacional de Rosario, Rosario, Argentina; ${ }^{4}$ Department of Epidemiology, UAB; ${ }^{5}$ Servicio de Reumatología, Hospital Privado Universitario de Córdoba; ${ }^{6}$ Sección de Reumatología, Servicio de Clínica Médica, Instituto Universitario, Hospital Italiano de Buenos Aires; ${ }^{7}$ Rheumatology Division, Faculdade de Medicina, Hospital das Clinicas HCFMUSP, Universidade de São Paulo; ${ }^{8}$ Facultad de Medicina y Ciencia, Universidad San Sebastián; ${ }^{9}$ Centro de Investigación Clínica de Morelia SC; ${ }^{10}$ Division of Rheumatology. University of Puerto Rico; ${ }^{11}$ School of Medicine, The University of Alabama at Birmingham; ${ }^{12}$ Centro Regional del Enfermedades Autoinmunes y Reumáticas (GO-CREAR)

\subsection{6/lupus-2019-Ism.289}

Background Classifying patients as having systemic lupus erythematosus (SLE) is critical for clinical trials and observational studies; although not designed for this purpose, criteria are also frequently used in clinical practice for early diagnosis. The SLICC 2012 criteria are more sensitive but less specific than the 1982/1997 ACR criteria. The refined 2018 EULAR/ ACR criteria differ from the other two sets as they require a positive ANA as the entry point; in addition, the clinical manifestations are clustered into weighted domains with the goal of maximizing the likelihood of an accurate classification of SLE, particularly of early disease.

The objective of the present study was to identify the distinct items of the clinical and immunological domains of the EULAR/ACR SLE classification criteria that differ in the time to criteria fulfillment when compared to the 1982/1997 ACR criteria in two multiethnic lupus cohorts.

Methods Patients from two multiethnic, multicenter cohorts, one from the US and the other from Latin America were included. For these analyses, EULAR/ACR items were evaluated to determine which clinical manifestations and/or laboratory parameters could be of help to achieve an earlier classification of patients. Categorical variables were compared using Chi-square or modified Fisher exact tests, as appropriate. The statistical analyses were performed using SAS software version 9.4.

Results Five-hundred and fifty-eight patients out of 640 from the US cohort and 956 out of 1047 from the Latin America cohort were included. Only $41(7.3 \%)$ and $71(7.4 \%)$ of patients achieved the 2018 EULAR/ACR criteria earlier in the US and Latin American cohorts, respectively. In turn, about one third of the patients in both cohorts achieved them later. Patients who accrued the 2018 EULAR/ACR earlier were more likely to have high anti-dsDNA titers and later earlier classification less likely to have mucocutaneous and joint manifestations; these data are depicted in table 1.

Conclusions When both cohorts were taken it into account, those patients who achieved the 2018 EULAR/ACR criteria 